DEESE, J., \& KRESSE, F. H. An experimental analysis of the errors in rote serial learning. Jounal of Experimental Psy chology, 1952,44, $199-202$.

GLA2E, J. A. The association value of non-sense syllables. Joumal of Genetic Psychology, 1928, 35, 255-269.

GLANZER, M., \& DOLINSKY, R. The anchor for the serial position curve. Joumal of Verbal Leaming \& Verbal Behavior, 1965, 4, 267-273. GLANZER, M., \& PETERS, S.C. Reexamination of the serial position effect. Journal of Experimental Psychology, 1962, 64, 258-266. HOVLAND, D. I. Experimental studies in rote-learning theory: II. Reminiscence with varying speeds of syllable presentation. Joumal of Experimental Psychology, 1938, 22, 338-353.
MaCRARY, J. W., \& HUNTER, W. S. Serial position curves in verbal learning. Science, 1953,117, 131-134.

RIBBACK, A., \& UNDERWOOD, B. J. An empirical explanation of the skewness of the bowed serial position curve. Joumal of Experimental Psychology, 1950, 40, 329-335. SLAMECKA, N. Human learning and memorySelected readings. New York: Oxford University Press, 1967.

\section{NOTE}

1. We express gratitude to Dr. Walter Makous for his many helpful suggestions, and to Mr. Michael Murray for assistance in the analysis of the data. This study was supported in part by the USPHS Training Grant No. ST01 GM 00666.

\title{
Temporal ROC curves and the psychological moment'
}

\author{
JONATHAN BARON, Mental Hcalth \\ Research Institute, The University of \\ Michigan, Ann Arbor, Mich. 48104
}

Subjects were asked to tell which of two points on a visual display moved first, and to indicate their confidence according to a six-category scale. One of the two points moved 32 msec before the other in all cases, but the order varied at random. ROC curves were straight lines on normal-normal paper. The results are inconsistent with the theory of a "psychological moment" but are consistent with the idea of "temporal noise" in perception.

Several classes of evidence (Stroud, 1955; White, 1963; Shallice, 1967) point to the existence of a quantal unit of psychological time which has been called the psychological moment. According to this moment theory, psychological time is divided into discrete, nonoverlapping intervals or moments. A specific implication of one form of this theory is that events occurring within the same moment are perceived as simultaneous. Only events falling in different moments are perceived as occurring at different times. The presumed moment should thus limit the ability of Ss to detect the order of occurrence of two stimuli. This limitation would hold, however, only when the $S$ is prevented from using cues other than the apparent times of occurrence of the stimuli, such as apparent motion (Thor, 1968) or apparent position (Békésy, 1967). While estimates of the psychological moment are never less than $50 \mathrm{msec}$, Ss can discriminate the order of two stimuli when their temporal separation is on the order of a millisecond as long as the use of these other cues is permitted.

We shall compare the moment theory to an alternative theory, which we shall call the noise theory. This theory holds that the time between the occurrence of a stimulus and its perception is somewhat variable. Failures to correctly discriminate the order of two stimuli may be ascribed to the variability of the times it takes to perceive the two stimuli. It will be assumed for simplicity, that the distribution of these times is normal.

Kristofferson (1967) has tested the moment theory in an experiment in which Ss were asked to say whether two stimuli were simultaneous or successive. The interval between the stimuli was varied from trial to trial. The critical data was a plot of the probability of saying that the stimuli were successive against the interval between the stimuli. According to the moment hypothesis, such a plot should be fit by a straight line. Although Kristofferson has fit such data with straight lines, others (Hirsch \& Sherrick, 1961) have fit similar data with normal ogives. A normal ogive would be predicted by the noise hypothesis described above. In fact, the two types of curves are difficult to distinguish on the basis of experimental data. An ogive is very close to a straight line over much of its length. Furthermore, if the duration of the moment were only slightly variable, the line predicted by the moment hypothesis would be rounded near probabilities of zero and one, and would look even more like a normal ogive.

The present experiment uses a different approach to distinguish between the moment theory and the noise theory. Like the experiments described above, the $S$ is asked to tell which of two stimuli came first But unlike the above experiments, the temporal separation between the stimuli is held constant, only their order being varied from trial to trial. Also, the $S$ is asked to indicate his confidence in each of his judgments. According to the theory of signal detection (Green \& Swets, 1966) such confidence ratings may be used to infer what the behavior of the $S$ would be if he were asked to vary his criterion or response bias for saying that one of the stimuli came first. The $S$ will be more likely to say that a given stimulus came first when the bias towards that response is high, or when the criterion for it is low. The data of interest to us is a plot of the probability of saying that one of the stimuli came first when it did (hits) against the probability of saying that it came first when it did not (false alarms) as the criterion or response bias is varied. Such a curve is known as the receiver-operatingcharacteristic ( $R O C$ ) curve.

According to the noise hypothesis, the ROC curve should resemble that obtained in other situations in which noise is also presumed to be the limiting factor affecting discriminability. We may apply the theory of signal detection to distributions of perception times for the two stimuli. According to this theory, the confidence ratings are taken to refer to a set of criteria based on a comparison of the probabilities of the two alternatives (one stimulus coming first or the other) given the perceptual experience of the S. The ROC curve should be bowed downwards when plotted in terms of probabilities, but linear when plotted in terms of the normal abscissae corresponding to the probabilities, i.e., on normal-normal coordinates.

According to the moment hypothesis, a "threshold" theory, only when the stimuli fall in different moments will their order be discriminable. Let us call the probability of the two stimuli ( $A$ and $B)$ coming in different moments with one of them (A) coming first, $\mathbf{p}\left(\mathbf{A}^{\prime}\right)$. Let us call the probability of the two stimuli falling within the same moment $\mathrm{p}(\mathrm{M})$. When the stimuli fall within the same moment, it is assumed that the S's guesses are independent of the stimulus conditions and that he has some response bias, $X$, for saying that $S$ timulus $A$ came first. This response bias is reflected in his confidence ratings. Thus the probability of a hit is $p(a / A)=p\left(A^{\prime}\right)+X p(M)$, where $a$ is the $S$ saying that $S$ timulus $A$ came first. On the other hand, the probability of a false alarm is $p(a / B)=X p(M)$. It follows that $p(a / A)=p(a / B)+p\left(A^{\prime}\right)$. Thus the ROC curve predicted by the moment hypothesis is a straight line when plotted in terms of probabilities, and is bowed upwards when plotted on normal-normal paper. Further, the only parameter involved in this equation is $p\left(\mathrm{~A}^{\prime}\right)$, which may be interpreted as the 




Fig. 1. ROC curves for six Ss. The abscissa is the $\mathrm{z}$ corresponding to the probability of saying that the right stimulus came first when the left stimulus came first. The ordinate is the $z$ corresponding to the probability of saying that the right stimulus came first when it did. Each S's results are plotted with reference to a separate origin.

proportion of trials in which the stimuli do not fall within the same moment and in which A comes first. Thus, the ROC curve would still be a straight line even if the duration of the moment were variable.

\section{METHOD}

The stimuli consisted of changes in position of two points of light plotted on an oscilloscope (Teletronics 561a). The CRT in this oscilloscope used an ultrashort persis tence phosphor (P-15), the light output of which decays to $0.1 \%$ of its maximum value in 50 microseconds. Three points were used in all. Each of these points was plotted once every millisecond. The two stimulus points were $13 \mathrm{~mm}$ to the right and to the left of the central fixation point. The two stimulus points were either $0.62 \mathrm{~mm}$ above or below the horizontal plane of the fixation point Both points moved either up or down on each trial, that is, from their position below the plane of the fixation point to thei position above, or vice versa. One point moved $32 \mathrm{msec}$ before the other, but the order of movement of the points was random from trial to trial. The points were plotted and the data collected by a PDP-5 computer. ${ }^{2}$ The $S$ sat at a distance of $1 \mathrm{~m}$ from the display in a darkened, sound. insulated booth.

Each trial was begun with a warning stimulus, an interruption of the entire display which lasted about $30 \mathrm{msec}$. The movement of the first point occurred $1 \mathrm{sec}$ later. The $S$ was then given as much time as he wanted to make his judgment. He was instructed to make his response by giving a digit from 1 to 6 . The digit 1 was to mean that he was quite sure that he had seen the left point move first, 6 that he was quite sure that he had seen the right point move first; the other four responses were to represent intermediate degrees of confidence, spread out in such a way that roughly equal numbers of responses were made with each digit. The $\mathrm{S}$ gave his response verbally. The $\mathrm{E}$ then typed the response on a teletypewriter, and the warning for the next trial occurred immediately. Each session consisted of 192 trials. An opportunity for practice was given before the beginning of the session. No feedback was given.

Six Ss of college age were paid for their services in the experiment. The data reported here are from the first eight sessions of each $\mathrm{S}$.

\section{RESULTS AND DISCUSSION}

The ROC curves, based on pooled data for the eight sessions, are shown in Fig. 1. The curves were plotted by considering all responses above a given digit as an estimate of what the S's responses would be with a given criterion, as described by Green \& Swets (1966). It can be seen that the curves are linear ${ }^{3}$ on normal-normal paper. If plotted in terms of probabilities, the curves are bowed downwards. Our data thus support the noise hypothesis, and fail to support the moment hypothesis. ${ }^{4}$

If it is true that the time at which an event is perceived is normally distributed with respect to its time of occurrence, some sort of temporal noise may be said to exist. What is the origin of this noise? As a first guess at a psychological explanation, it appears that the noise has something to do with the attention of the $S$. The $E$ has found that he can control his perception of which point moves first-when the two points in fact move at the same time and his gaze is fixed on the fixation point-by attending to one point or the other. James (1890) discusses at length some of the early evidence for the relation between attention and time of perception, the "prior entry" problem. The general finding was that a stimulus seemed to come earlier if the $S$ attended to it. Fluctuations of attention between the two stimuli could account for the variability in time of perception. Schmidt \& Kristofferson (1963) have made a similar proposal. Their view of attention, however, is an all-or-none phenomenon: Attention is either focused on a stimulus or not, at a given point in time, and cannot be focused on more than one stimulus at once. Our results, however, seem to force us in to regarding attention as a more flexible entity, insofar as it may be invoked to explain them.

\section{REFERENCES}

BÉKÉSY, G. von. Sensory inhibition. New York: McGraw-Hill, 1967.

GREEN, D. M., \& SWETS, J. A. Signal detection theory and psychophysics New York: John Wiley \& Sons, 1966.

HIRSCH, I. J., \& SHER RICK, C. E., JR. Perceived order in different sense modalities. Journal of Experimental Psychology, 1961, 62, 423-432.

JAMES, W. Principles of psychology. New York: Henry Holt, 1890.

KRISTOFFERSON, A. B. Successiveness discrimination as a two-state, quantal process. Science. $1967,158,1337-1339$

SCHMIDT, M. W., \& KRISTOFFERSON, A. B. Discrimination of successiveness: A test of a model of attention. Science, 1963, 139 112-113.

SHALLICE, T. Temporal summation and absolute brightness thresholds. British Journal of Mathematical \& Statistical Psychology, 1967, 20,129-162.

STROUD, J. M. The fine structure of psychological time. In H. Quastler (Ed.), Information theory in psychology. New York: Free Press, 1955

THOR, D. H. Observer strategies in dichoptic viewing of successive stimuli. Psychonomic Science, 1968, 12, 41-42.

WHITE, C. T. Temporal numerosity and the psychological unit of duration. Psychological Monographs, 1963, (Whole No. 575). NOTES

1. This work was supported by NSF Grant GB 6093. The author is grateful to William $R$. Uttal in whose laboratory this work was done.

2. Evoked potentials were also recorded during the experiment. These data will be reported in the future.

3. For one of the Ss (JG) the ROC curve appeared to be bowed upwards, as predicted by the moment theory-although it was still bowed downwards when plotted in terms of probabilities. On close inspection, it was found that this $S$ seemed to use Response Categories 3 and 4 in reverse-a higher proportion of $4 \mathrm{~s}$ being used when the left stimulus came first. The curve shown for this $S$ has been plotted by combining the results of Categories 3 and 4 . This $S$ reported that she often did not see one of the stimuli. When this happened she would respond " 3 " or " 4 ." Because of this inability to order the categories properly, and the suspicion of artifact, it is felt that these results cannot be taken seriously.

4. Additional support for the noise hypothesis is provided by two experiments which the author carried out on himself. In the first, it was shown that $\mathrm{d}^{\prime}$ is a linear function (passing through the origin) of the interval between the two stimuli (8. 16 , or $32 \mathrm{msec}$. In the second, Stimuli $A$ and $B$ were either simultaneous, or A preceded B by $32 \mathrm{msec}$. In this case, $d^{\prime}$ was about half of what it was for the A-B, B-A discrimination described above. 